\title{
Free Space Optical Interconnects Security Aspects of Future High Speed Technology
}

\author{
Priyanka Jangir \\ M.tech. Student \\ Dehradun Institute of \\ Technology Dehradun, India
}

\author{
Bhupendra Suman \\ Scientist-'E' \\ Defense Electronics Application \\ Lab. DRDO, Dehradun, India
}

\author{
Rashmi Chaudhary \\ Lecturer ECE dept. \\ Dehradun Institute of \\ Technology Dehradun, India
}

\begin{abstract}
Free space optical (FSO) communication involves the use of optical or laser beams to send the information through the atmosphere. The key towards our vision is the use of FSO technology which in contrast to traditional wireless of RF technologies has lower interference footprint, can support longer range and offers higher bandwidth. In this paper we review the recent advances made in the field of data security for the FSO link along with certain aspects of FSO which make this technology most secure than the other existing technologies and discuss future research direction needed to bring FSO to the realm of practice.
\end{abstract}

\section{Keywords}

Atmospheric structure parameter, FSO, BER, Scintillation, Terescope

\section{INTRODUCTION}

In today's world broadband communication is faster and more accessible than ever before. Over 16 million people pay a premium to access the data using high speed connection and many more want to join them but can't, due lack of infrastructure, last mile challenges and cost competitive issues. One of the solutions to above problems is FSO communication which can offer high data rates but also the same flexibility as wireless microwave systems. FSO based systems are beyond these challenges and can provide us with powerful new solutions. Using this technology, connections can be made between existing systems, wireless, wire line, fiber optic or satellite using invisible beam of light resulting in a very fast wireless broadband network with major advantages over other wireless systems. Some of the advantages are: there is no spectrum licensing, reduction in deployment time and cost, interference free transmission and nearly impossible interception making FSO most secure wireless technology. Further the short wavelengths allow a more compact design of components and lower power consumption than microwave systems do, which is very important for aeronautical and space systems. Also, it is resistant of spying due to the very small convergence angle of the beam. All this presents free space optical communications to be an attractive alternative for many kinds of wireless communication scenarios. Future applications may include networks of HAPS (high altitude platforms, hovering in the stratosphere), UAVs (unmanned aerial vehicles) and airplanes, whereas all this aeronautical objects can be optically connected amongst each other and/or additional satellites [1].

Along with various advantages of FSO over other networks there some common misconceptions regarding the data security in the network as the medium for the transmission of the information is free space or the open environment which is accessible to everyone so we need to assure the security of our information transferred through the network. In our work we focus on the security of the link and various factors which make it practically impossible to hack the data from the link. In this paper an attempt has been made to elaborate information on certain aspects of this technology and steps towards making this technology more robust and secure for fulfilling the need of future high speed communication systems.

The remainder of this article is organized as follows. In section 2 we give a basic view of the FSO architecture. Section 3 includes the theory of link performance in FSO and section 4 and 5 describe the methods used in an FSO system to tackle various natural and human generated disturbances. And finally the paper is concluded in section 6 .

\section{FSO COMMUNICATION SYSTEM}

A free space optics communication system (fig.1) consists of a small laser source that can be directly modulated in intensity at fairly high data rates; a beam shaping and transmitting telescope lens to transmit the laser beam through the atmosphere toward a distant point; a receiving lens or telescope to collect and focus the intercepted laser light onto a photo detector; and a receiver amplifier to amplify and condition the received communication signal. The transmitted laser beam passes through the atmosphere and can be absorbed, scattered or displaced, depending on atmospheric conditions and on the wavelength/line width of the laser source [2].The block diagram of FSO link as

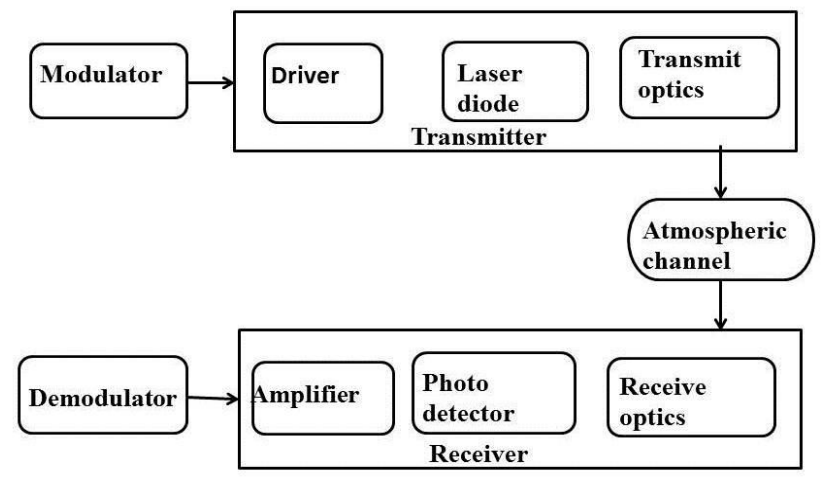

Fig 1: Block diagram of FSO

A typical FSO transmitter (LASER, VCSEL or LED) forms a cone shaped volume in 3 dimensions (Fig. 2) in which a potential receiver equipped with a photo detector can receive the signal. The exact shape of this cone is determined by the transmission power (for range) and divergence angle of the source. A LASER has the smallest (in micro radian range) and an LED has the widest (a few hundred milli-radians) divergence angle of the three types of transmitters. FSO can operate in large swathes of unlicensed spectrum reaching speeds up to 1 Gbps. Additionally, FSO transceivers have much smaller form factors, are less power-consuming (100 microwatts for $10-100 \mathrm{Mbps}$ ), very reliable (lifetime of more 
than 10 years), cheap and offer highly directional beams for spatial reuse and security [3].

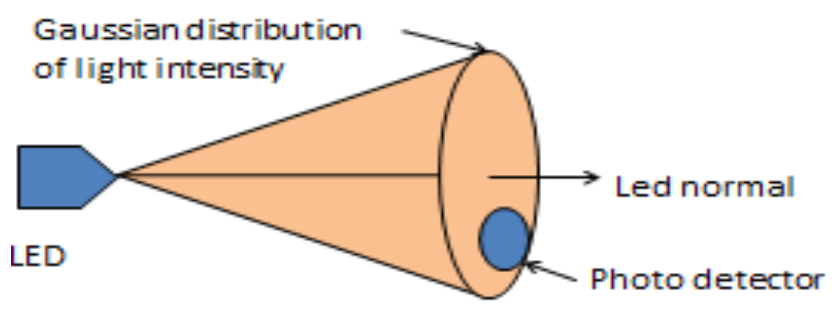

Fig. 2: Gaussian distribution of light intensity at the receiver plane

\subsection{FSO Architectures}

FSO technology has proven itself in other applications, particularly those requiring a tactical, point-to-point link. FSO's greatest success so far has come from the LAN/campus connectivity market. Such applications could include a link between a newsroom and a broadcasting station, or a dedicated link between two high-traffic nodes in a large building complex. Thus, to maintain quality of service for a particular customer, the distance from that customer's location to the nearest hub may have to be shortened.

Three main FSO architectures have been used so far [4].

- Point to point architecture is a dedicated connection that offers higher bandwidth but is less scalable.

- Mesh architecture may offer redundancy and high reliability with easy node addition but restrict distances more than the other options.

- Point to multipoint architecture offers cheaper connections and facilitates node addition but at the expanse of lower bandwidth than the point to point option.

For the mesh and point to multipoint the number of telescope units used in the system is more the one.

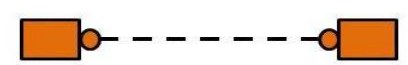

(a).Point to point

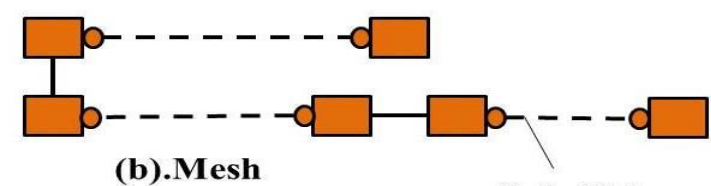

(b).Mesh

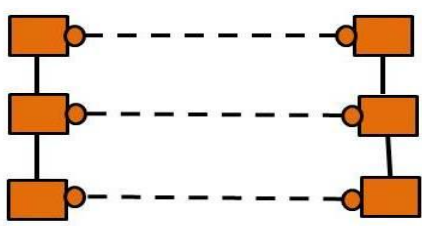

Optical link

(c).Point to multipoint

Fig. 3: FSO architectures

\section{THEORY OF OPTICAL LINK PERFORMANCE}

The FSO link performances can be determined by several parameters including geometrical loss, link margin, received power and BER. There are two parameters to evaluate the FSO link performances which are the received power and BER. Theoretically, the basic communication principle stated that received power must be less than transmitted power, $\mathrm{PR} \leq \mathrm{PT}$, according to [5]:

$$
P_{R}=P_{T} \text {-Total-losses }
$$

Where $P_{R}(\mathrm{dBm})$ is the received power, $P_{T}(\mathrm{dBm})$ is the transmitted power. According to [6], total losses in a FSO communication system would cover all the losses caused by the atmospheric phenomena, $L_{A T M}(\mathrm{~dB})$ which can be calculated as in Eq. (5), geometrical loss, $L_{G E O}(\mathrm{~dB})$ and system loss, $L_{S Y S}(\mathrm{~dB})$. Therefore, the new equation for FSO received power is as in Eq. (2):

$$
P_{R}=P_{T c o m b}-L_{A T M}-L_{G E O}-L_{S Y S}
$$

The total transmitted power can be obtained in Eq. (3):

$$
P_{\text {Tcomb }}=P_{T}+10 \log _{10}\left(N_{T}\right)
$$

Where, $N_{T}$ is the number of transmitter lenses on a single FSO unit. Geometrical loss and system loss are the internal losses occurred within the FSO transceiver. Both losses are fixed on all FSO link and cannot be neglected. $L_{S Y S}$ Is manufacturer defined; meanwhile in [6] $L_{G E O}$ can be calculated as in Eq. (4):

$$
L_{G E O}=-10 \log _{10}\left[\frac{A_{R T o t a l}}{\pi(l \theta)^{2}}\right]
$$

$\ell(\mathrm{km})$ is the distance of the optical path where the laser beams travel and $\theta$ (mrad) is the divergence angle which is the angle of the cone of light emitted from the transmitter. Meanwhile, $A_{\text {RTotal }}\left(\mathrm{m}^{2}\right)$ is the total area of the receiver apertures on a single FSO unit.

According to Beers-Lambert Law, the atmospheric losses for any laser power is in a form of exponential equation of:

$$
L_{A T M}=e^{n l}
$$

Where $\ell(\mathrm{km})$ is the transmittance range of the laser and $\sigma$ is the typical attenuation coefficients $(0.1$ for clear air)[6]. 
The robustness of the design of any optical communication system can be effectively verified by critically applying performance checks on the system. The evaluation criteria should provide a precise determination and separation of dominant system limitations, making them crucial for the suppression of propagation disturbances and performance improvement. The Bit Error Rate (BER) of an optical link is the most important measure of the faithfulness of the link in transporting the binary data from transmitter to receiver. The BER quantifies the rate of errors and is defined as the probability of an error occurring per transported bit. The bit error rate takes the simple form

$$
\mathrm{BER}=\frac{1}{2} \operatorname{erfc}\left(\frac{Q}{\sqrt{2}}\right)
$$

Where $Q$ is quality factor and 'erfc' denotes the complementary error function [7].

The above equations represent some performance analyzing parameters of a basic optical link. These are some of the parameters which can be altered and readjusted at the transmitters end. One value can compromised in terms of another value as per the need of the link. But if we talk in terms of the security of any communication link than there are other factors also on which the reliability of the link depends. For the FSO link the total security of the link depends on the laser light beam which is used to transmit the signals. Here we discuss various limiting factors of the free space communication link and how FSO comes up crossing all the hindrance in the path of the link.

\section{ATMOSPHERIC EFFECTS ON FSO LINK}

The actual transmission rates are weather dependent as the atmospheric attenuation is variable and hard to predict. The link may not work well in locations where the atmosphere is affected by conditions such as fog, storm, scintillation etc.

\subsection{FSO and fog}

Fog substantially attenuates visible radiation and it has a similar effect on the near infrared wavelengths that are employed in the FSO system $(1550 \mathrm{~nm})$. Note that the effect of fog on the free space optical wireless radiation is entirely analogous to the attenuation and fades suffered by the RF wireless system due to rain fall. Similar to the case of rain attenuation with RF wireless fog attenuation is not a 'showstopper' for FSO (signals block out on the television during heavy rain) because the optical link can be engineered such that for a large fraction of time an acceptable power is received even in presence of heavy fog. FSO optical wireless based communication system can be enhanced to yield even greater availabilities [8].

\subsection{FSO and scintillation}

Atmospheric scintillation can be thought of as changing light intensities in time and space at the plane of a receiver detecting the signal from a transmitter at a distance. When the beam is scintillated, photons of light are temporally steered by pockets of air in random direction. This is the same atmospheric effect what causes stars to appear to twinkle at night. The received signal level at the detector fluctuates due to thermally induced changes in the index of refraction of the air along the transmit path. The index changes cause the atmosphere to act like a collection of small prisms and lenses that deflect the light beam into and out of the transmit path. The time scale of these fluctuations is about the time it takes a volume of air the size of the beam to move across the path and therefore is related to wind speed. For the case of free-space optics, which implies horizontal path propagation and therefore stronger scintillation, the distribution tends to be more exponential. One parameter that is often used as a measure of the scintillation strength is the atmospheric structure parameter which is denoted as $\boldsymbol{C}^{\boldsymbol{n}^{2}}$. This parameter, which is directly related to wind speed, roughly measures how turbulent the atmosphere is [9].

To overcome the scintillation effects automatic gain control mechanism is used and also clock recovery phase lock loop time constant eliminates the effects of scintillation and jitter transference [8].

\subsection{FSO and solar interference}

Solar interference in FSO systems operating at $1550 \mathrm{~nm}$ can be combatted in two ways. Firstly by using a long pass optical filter window to block all optical wavelengths below $850 \mathrm{~nm}$ from entering the system and secondly by using optical narrow band filter preceding the receiver detector to filter all but the wavelength actually used for communication. To handle the off axis solar energy two spatial filters are used. These sub systems allow FSO links to operate unaffected by solar interference that is more than 1.5 degrees off axis [8].

\section{FSO AND UNAUTHORIZED HUMAN INTERRUPTION}

FSO is being used in the field of disaster recovery and temporary applications along with inter satellite and under water communication. Currently FSO is used by businesses man who own buildings with line of sight. For them security and uninterrupted reception is very important.

\subsection{Security in FSO}

The tightly collimated FSO beam ensures that the signal energy is focused on the receiving unit making interception of the beam extremely difficult the major reason behind this is the absence of the side lobes. Pick off a signal through random scattering effects is practically impossible. Over long distances temperature changes between pockets of air which can cause the beam to scatter slightly. This is known as scintillation. These air pockets are in permanent motion and so the boundaries between various pockets are also constantly changing. Because scintillation is caused by laser light passing through these boundaries of the neighboring air pockets the path of twinkling photons is always changing randomly. These changes from scintillation last only for fraction of a second. Since the angle of scintillation is random and changes are very fast, it is very difficult to forecast a specific spot of intercepting the signal.

\subsection{Need for a complete uninterrupted link}

FSO laser beam cannot be detected with spectrum analyzer or RF meters because these beams are narrow and invisible, making them harder to intercept and track. For an optical beam the signal interruption is possible only by placing a detector in the path or in the focusing area of the beam. If someone attempts to intercept a signal by placing a detector in the path of the beam it will be blocked and communication will be terminated. The only way to pick off the narrow beam path is from the location behind the building on which the receiving unit is installed. To prevent this unlikely event beam shielding can be done and there are two ways to do so (a) install the receiver against the wall which is twice as wide as radius of the FSO beam and (b) if the link is installed on a rooftop or an open surface it is possible to place a covering or shield behind the receiver (twice as large as beam diameter). 


\subsection{Signal decoding prevention from intruder}

All switching and routing security features existing in a wire line infrastructure can be carried over to the terescopes (device used for beam focusing in FSO). Since the terescope systems are encryption devices that are added to the link to offer additional security. The terescope passively retransmit an encoded bit stream, making outside signal decoding even more difficult [10].

\section{CONCLUSION}

A brief survey of fundamental security aspects has been presented in this paper. This brief survey focuses on outdoor FSO static optical links and describes the methods by which FSO emerges as a strong opponent to the RF communication in terms of link utilization and security. The advantage of FSO results from its high frequency, coherence and low divergence which lead to a high information carrying link.

\section{REFERENCES}

[1] Florian Moll, "Conception and development of an adaptive optics test bed for free-space optical communication," Master's Thesis, Institute for Measurement Systems and Sensor Technology (TUM).

[2] Dennis Killinger, Free Space Optics for Laser Communication Through the Air. Optics \& Photonics
News, Optical Society of America Optics \& Photonics News, October 2002.

[3] M. Bilgi and M. Yuksel, "Packet-Based Simulation for Optical Wireless Communication," IEEE Work- shop on Local and Metropolitan Area Networks (LANMAN), Long Branch, New Jersey, May 2010.

[4] www.fiberwork.net

[5] I. D. A. Singapore, A trial-based study of free-space optics systems in Singapore, Info-Communications Development Authority of Singapore (Ida), Singapore 2002.

[6] N. Haedzerin Md Noor, Ahmed Wathik Naji and Wajdi Al-Khateeb, Performance analysis of a free space optics link with multiple transmitters/receivers, Iium Engineering Journal, Vol. 13 No. 1, 2012 Pg.49-58.

[7] G. Soni, Jagjit M. Singh , Impact of Beam Divergence on the Performance of Free Space Optical System, IJSRP, Volume 2, Issue 2, February 2012, ISSN 2250 3153.

[8] www.fsona.com

[9] S. Bloom The Physics of Free Space Optics, AirFiber Inc.2 May 2002

[10] www.lightpointe.com 